\title{
Acute Care at Home During the COVID-19 Pandemic Surge in Boston
}

J Gen Intern Med 36(11):3644-6

DOI: $10.1007 / \mathrm{s} 11606-021-07052-5$

(C) Society of General Internal Medicine 2021

\section{INTRODUCTION}

The COVID-19 pandemic has severely strained hospital systems, particularly in hotspot regions. The predominant national response has been to build tent hospitals in parking lots and parks, to convert large conference centers to field hospitals, or to bring floating hospitals to aide overwhelmed infrastructure. Sadly, most of these high-cost interventions have remained vastly underutilized. ${ }^{1}$

A different approach to optimizing hospital capacity is to provide acute care at home as a substitute for traditional hospitalization. "Home hospital" care includes a physician, nurse, and paramedic team that delivers advanced infusion, respiratory therapies, lab and imaging diagnostics, and continuous monitoring at home. A patient receives twice daily nurse or paramedic visits and a daily physician visit, coupled with $24 / 7$ response capability. Conditions include those typical of a general medicine ward: infections and exacerbations of heart failure, asthma, and chronic obstructive pulmonary disease. In several randomized controlled trials, it has better or similar quality, safety, and experience outcomes compared to traditional hospital care. ${ }^{3}$

Whether during an infectious pandemic a home hospital would continue to provide a high level of care while creating inpatient capacity was unknown.

\section{METHODS}

We performed a retrospective analysis of our home hospital program operating within 5 miles of 2 sites in Boston, Massachusetts: Brigham and Women's Hospital and Brigham and Women's Faulkner Hospital. Detailed operations have been described previously. ${ }^{3}$ During COVID-19, home hospital programs chose to continue treating their standard diagnoses or to add COVID-19 to their inclusion criteria. Due to severe personal protective equipment availability constraints, our program chose the former.

Received March 27, 2021

Accepted July 20, 2021

Published online August 6, 2021
To evaluate home hospital's capacity creation, we included patients home hospitalized during pandemic surge conditions, beginning March 15, 2020 (when Massachusetts state of emergency restrictions took effect) until the surge ended on June 18, 2020 (defined as $<30$ patients hospitalized with COVID-19). We analyzed patient characteristics and outcomes through medical record abstraction. The Mass General Brigham IRB approved this study.

\section{RESULTS}

Over 95 days, we cared for 65 acutely ill patients at home, amounting to 419 bed-days, or $5 \%$ of all medicine patients without COVID-19 and $15.4 \%$ of all patients with COVID-19 admitted in our catchment area (Fig. 1). During this time, our program was staffed clinically each day by 1 physician, $1-2$ nurses, and 1 mobile integrated health paramedic and had a mean midnight census of 3.7 .

Patients were $57 \%$ female, $37 \%$ White, and 39\% partnered (Table 1). Median age was 66 (interquartile range, 34) and $75 \%$ primarily spoke English. We most commonly cared for infection (59\%) and heart failure exacerbation (22\%). Length of stay was 5.0 days (interquartile range, 4.1 ); escalation rate was $3.1 \%$. Most (65\%) were discharged without services, $12.3 \%$ were readmitted within 30 days, and $13.8 \%$ presented to the emergency department within 30 days.

\section{DISCUSSION}

We found that despite a COVID-19 pandemic surge, a small home hospital team created substantial inpatient capacity while delivering high-quality acute care at home. To put this in context, the field hospital established to create capacity for Boston's acute care infrastructure served 394 patients (mean 57 years old, $29 \%$ female, $19 \%$ White, mean of 6 outpatient medications; length of stay 8.3 days, $6.6 \%$ escalation rate), required onboarding 124 providers and 331 nurses, and cost $\$ 29.8$ million over a similar time period-more than $\$ 75,000$ per patient. ${ }^{4,5}$ Traditional hospitalization typically costs about $\$ 15,000$ per patient, and home hospital care is about 38\% less expensive than hospital care. ${ }^{3}$

Our work builds on others who demonstrated successful surge capacity creation with home hospital programs that took care of patients with COVID-19. ${ }^{6}$ Our work demonstrates that home hospital serves an important function whether or not it 


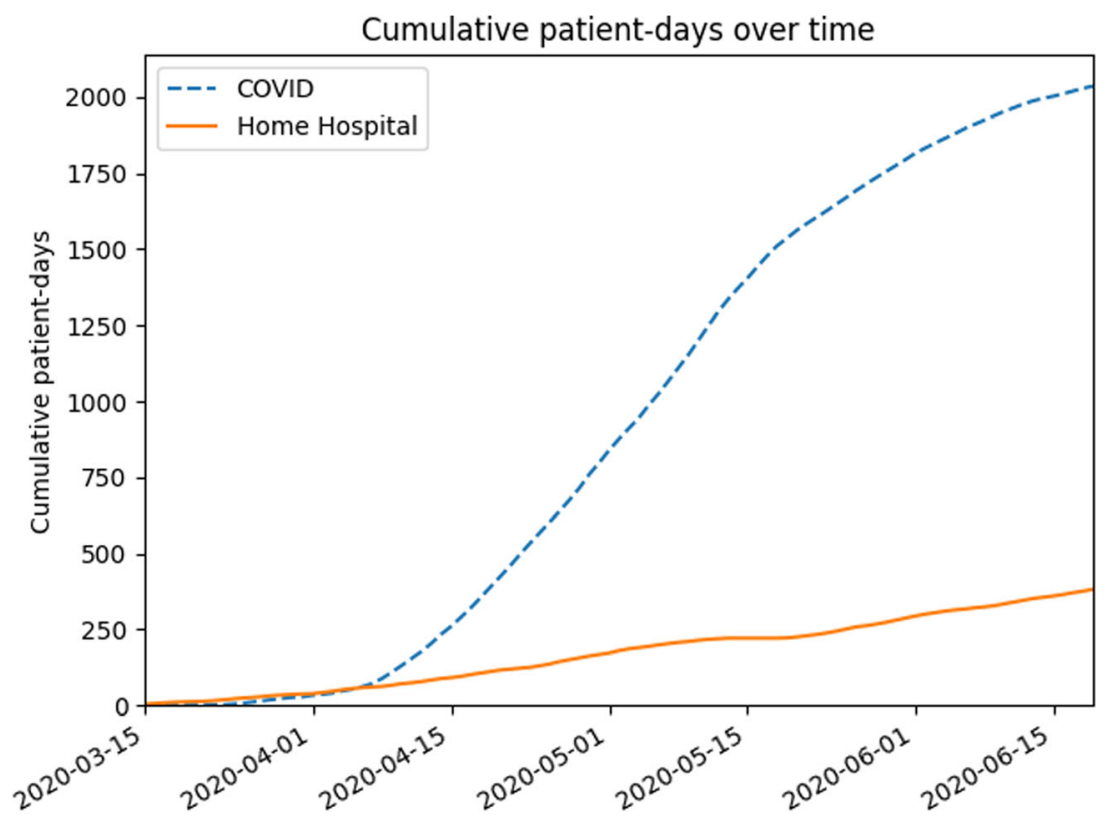

Figure 1 Bed requirements for patients with COVID-19 and home hospital bed capacity creation.

Table 1 Patient Characteristics and Outcomes

\begin{tabular}{ll}
\hline \hline & $\begin{array}{l}\text { Home hospital patients } \\
(\boldsymbol{n}=\mathbf{6 5})\end{array}$ \\
\cline { 2 - 2 } & $\boldsymbol{n ( \% )}$ \\
\hline Baseline characteristics & \\
Median age (IQR), years & $66(34)$ \\
Female sex & $37(56.9)$ \\
Race/ethnicity & $24(36.9)$ \\
White & $15(23.1)$ \\
Black & $12(18.5)$ \\
Hispanic or Latino & $7(10.7)$ \\
Other & $4(6.2)$ \\
Unknown & $25(38.5)$ \\
Partner status & $21(32.3)$ \\
Partnered & $17(26.2)$ \\
Single & $2(3.1)$ \\
Separated & $49(75.4)$ \\
Unavailable & $12(18.5)$ \\
Primary language & $4(6.2)$ \\
English & \\
Spanish & $30(46.2)$ \\
Other & $27(41.5)$ \\
Insurance & $7(10.8)$ \\
Medicare & $1(1.5)$ \\
Private & \\
Medicaid & $18(27.7)$ \\
None & $16(24.6)$ \\
Education & $15(23.1)$ \\
Less than high school & $15(23.1)$ \\
Graduated high school & $1(1.5)$ \\
< 4-year college & $9(13.8)$ \\
4-year college & \\
>4-year college & $29(44.6)$ \\
Unknown & $16(24.5)$ \\
Employment & $12(18.5)$ \\
Retired & $7(10.8)$ \\
Employed & $1(1.5)$ \\
Not employed & \\
Disabled & $32(49.2)$ \\
Unknown & $27(41.5)$ \\
Cigarette smoking & $4(6.2)$ \\
Never smoker & $3(4.6)$ \\
Former smoker & \\
Current smoker & \\
Unknown & \\
Admitting diagnoses & $(c 0)$ \\
\hline
\end{tabular}

Table 1. (continued)

\begin{tabular}{|c|c|}
\hline & \multirow{2}{*}{$\begin{array}{l}\text { Home hospital patients } \\
(n=65) \\
n(\%)\end{array}$} \\
\hline & \\
\hline Skin/soft tissue infection & $15(23.1)$ \\
\hline Heart failure & $14(21.5)$ \\
\hline Other infection & $13(20.0)$ \\
\hline Complicated urinary tract infection & $10(15.4)$ \\
\hline Other & $7(10.8)$ \\
\hline Diabetes & $3(4.6)$ \\
\hline Chronic obstructive pulmonary disease & $3(4.6)$ \\
\hline \multicolumn{2}{|l|}{ Code status } \\
\hline Full code (confirmed or presumed) & $46(70.8)$ \\
\hline Do not resuscitate/do not intubate & $19(29.2)$ \\
\hline Admitted to hospital in past 6 months & $38(58.5)$ \\
\hline $\begin{array}{l}\text { Visited emergency department in past } 6 \\
\text { months }\end{array}$ & $37(56.9)$ \\
\hline Mean outpatient medications $(95 \% \mathrm{CI})$ & $8.2(6.9-9.4)$ \\
\hline \multicolumn{2}{|l|}{$\begin{array}{l}\text { Mean outpatient medications ( } 95 \% \text { C }) \\
\text { Comorbidities }\end{array}$} \\
\hline Asthma & $14(21.5)$ \\
\hline Coronary artery disease & $8(12.3)$ \\
\hline Heart failure & $14(21.5)$ \\
\hline Cancer & $7(10.8)$ \\
\hline Chronic kidney disease & $15(23.1)$ \\
\hline Diabetes & $22(33.8)$ \\
\hline Hypertension & $43(66.2)$ \\
\hline Chronic obstructive pulmonary disease & $6(9.2)$ \\
\hline \multicolumn{2}{|l|}{ Patient outcomes during the acute episode } \\
\hline Median length of stay (IQR), days & $5.0(4.1)$ \\
\hline IV medications & $51(78.5)$ \\
\hline Patients with PT/OT visits & $7(10.8)$ \\
\hline Patients with specialist consults & $7(10.8)$ \\
\hline Patients with imaging orders & $3(4.6)$ \\
\hline Median laboratory orders (IQR) & $3(8)$ \\
\hline Escalation $^{\mathbf{2}}$ & $2(3.1)$ \\
\hline \multicolumn{2}{|l|}{ Discharge disposition } \\
\hline Routine & $42(64.6)$ \\
\hline Home health & $22(33.8)$ \\
\hline Home hospice & $1(1.5)$ \\
\hline \multicolumn{2}{|c|}{ Patient outcomes 30 days after the acute episode } \\
\hline 30-day readmission & $8(12.3)$ \\
\hline 30-day emergency department presentation & $9(13.8)$ \\
\hline Primary care visit $\leq 14$ days after discharge & $10(15.4)$ \\
\hline
\end{tabular}

CI confidence interval, IOR interquartile range ${ }^{a}$ Return to the hospital for continued acute care 
treats patients with COVID-19. In addition, a home hospital program may enable care delivery for patients who would otherwise defer care during a pandemic.

Our analysis has limitations, all limiting generalizability: our patients were recruited at only 2 sites, a small rotating cohort of clinicians cared for the patients (4 doctors, 7 nurses, and 3 paramedics), and the pandemic conditions in Boston may not have occurred elsewhere.

On November 25, 2020, the Centers for Medicare and Medicaid Services (CMS) issued the Acute Hospital Care at Home waiver that creates a payment mechanism for home hospital. Since then, over 130 hospitals have taken up this care model, demonstrating significant scalability. This model could have an important impact on the ongoing response to the pandemic and future capacity creation for US acute care. Large multisite studies will help to further delineate home hospital's role.

David Michael Levine, MD MPH MA ${ }^{1,2}$

Henry Mitchell ${ }^{1}$

Nicole Rosario, $\mathrm{MS}^{1}$

Robert B. Boxer, $M D P h D^{1,2}$

Charles A. Morris, MD MPH ${ }^{1,2}$

Kathryn A. Britton, MD MPH ${ }^{2,3}$

Jeffrey L. Schnipper, MD MPH ${ }^{1,2}$

${ }^{1}$ Division of General Internal Medicine and Primary

Care, Brigham and Women's Hospital,

Boston, MA, USA

${ }^{2}$ Harvard Medical School,

Boston, MA, USA

${ }^{3}$ Division of Cardiovascular Medicine, Brigham and Women's Hospital,

Boston, MA, USA

Corresponding Author: David Michael Levine, MD MPH MA; Harvard Medical School, Boston, MA, USA (e-mail: dmlevine@bwh. harvard.edu).

Author Contribution - Levine had full access to all of the data in the study and takes responsibility for the integrity of the data and the accuracy of the data analysis.
- Study concept and design: Levine.

- Acquisition, analysis, or interpretation of data: all authors.

- Drafting of the manuscript: Levine.

- Critical revision of the manuscript for important intellectual content: all authors.

- Statistical analysis: Mitchell.

- Administrative, technical, or material support: Mitchell, Rosario.

- Study supervision: Schnipper.

\section{Declarations:}

Conflict of Interest: David Levine: Dr. Levine receives funding from Biofourmis for an investigator-initiated artificial intelligence study on deterioration of home hospital patients and home hospital software, separate from the present work. He also receives funding from IBM, separate from the present work.

Jeffrey Schnipper: Dr. Schnipper is the recipient of funding from Mallinckrodt Pharmaceuticals for an investigator-initiated study of opioid-related adverse drug events in post-operative hospitalized patients.

All other authors: None.

\section{REFERENCES}

1. Nelson B. Too little or too much? Missing the Goldilocks zone of hospital capacity during covid-19. BMJ. 2020;369. https://doi.org/10.1136/bmj. $\mathrm{m} 2332$

2. Leff B, Burton L, Mader SL, et al. Hospital at home: feasibility and outcomes of a program to provide hospital-level care at home for acutely ill older patients. Ann Intern Med 2005;143(11):798-808. Accessed 15 Feb 2016. http://www.ncbi.nlm.nih.gov/pubmed/16330791

3. Levine DM, Ouchi K, Blanchfield B, et al. Hospital-level care at home for acutely ill adults a randomized controlled trial. Ann Intern Med 2020;172(2):77-85. https://doi.org/10.7326/M19-0600

4. Baughman AW, Hirschberg RE, Lucas $\mathbf{L J}$, et al. Pandemic care through collaboration: lessons from a COVID-19 field hospital. J Am Med Dir Assoc 2020;21(11):1563-1567. https://doi.org/10.1016/j.jamda.2020.09.003

5. Kavanaugh K. Field hospitals to return to Mass.; the cost and how they were utilized in the spring. Boston 25 News. Published 2020. https:// www.boston25news.com/news/25-investigates/25-investigates-field-hospitals-return-mass-cost-how-they-were-utilized-spring/ VVFKFN7GFBFKNOYWF57TGMO2GQ/

6. Sitammagari K, Murphy S, Kowalkowski M, Chou SH, Sullivan M, Taylor S, Kearns J, Batchelor T, Rivet C, Hole C, Hinson T, McCreary P, Brown R, Dunn T, Neuwirth Z MA. Insights from rapid deployment of a "virtual hospital" as standard care during the COVID-19 pandemic. Ann Intern Med. 2020;0(0):null. https://doi.org/10.7326/M20-4076

Publisher's Note Springer Nature remains neutral with regard to jurisdictional claims in published maps and institutional affiliations. 